\title{
WORLD WIDE WEB
}

\section{Accident and emergency medicine-making waves on the internet}

\author{
John M Ryan, Clive Baldock, Ralph Lawson-Smith
}

\begin{abstract}
The internet is a communications and information tool which has recently entered the world of accident and emergency (A\&E) medicine. It is a worldwide instrument facilitating the dissemination of ideas and clinical information in the specialty. It is being embraced by all disciplines involved in A\&E medicine. Part I introduces the internet to those in $A \& E$ medicine unfamiliar with this technology. It describes the varied resources of the internet in A\&E medicine and speculates on its future role. Part II supplies the reader with the necessary information to get on-line and explains some of the more technical aspects of the internet. (F Accid Emerg Med 1997;14:392-397)
\end{abstract}

Keywords: internet; accident and emergency medicine

Royal Sussex County Hospital, Eastern Rd, Brighton, UK

J M Ryan

Centre for Medical and Health Physics, Queensland University of Technology, Brisbane, Australia C Baldock

Westmead Hospital, Sydney, Australia R Lawson-Smith

Correspondence to: Mr John M Ryan, Accident and Emergency Department, Royal Sussex County Hospital, Eastern Rd, Brighton BN2 5BE; email: J.M.Ryan@sussex.ac.uk

Accepted for publication 28 May 1997 with the splitting of the Advanced Research Projects Agency network into a military and a research network. New networks were developed for the computer sciences and for academic communities. In 1986 the National Science Foundation in the United Sates established five super computer centres which formed a backbone for the internet as we have come to know it today. Other countries simultaneously developed computer networks which soon started to link up. This was followed by a massive increase in the numbers of operators of linked networks coming on-line, and the introduction of the private and commercial sector.

In the early 1990s the power of the internet as a resource tool for medicine was recognised. Initially access to the internet was gained through the UNIX system, an arduous communications system enabling communication between a distant computer and one permanently connected to the internet. The development of the world wide web (WWW) by the Cern Institute in Geneva in 1989 was a major milestone in the evolution of the internet and attracted the medical profession to the internet, recognising its power as a communications tool and as an access tool to the "information superhighway." The computer protocol used by the Web, known as hypertext markup language, has facilitated easy presentation of text, graphs, tables, moving images, and even sound on one's personal computer.

In the United Kingdom, facilities were provided by such academic groups as JANET (the joint academic network) and MAILBASE, the UK's major electronic mailing list service. In 1995 hospitals started to publish on the internet in the UK. Brighton Healthcare NHS Trust was the first trust to publish information and included a linked web page on its $A \& E$ department. ${ }^{1}$ Others have since followed suit.

The years 1988 to 1994 saw an increase of $4700 \%$ in registered internet hosts! The number of doctors using the internet has increased dramatically as the medical profession has begun to realise its potential for communication and continuing medical education, and its role in clinical support. Doctors working in $\mathrm{A} \& \mathrm{E}$ and in the emergency services have identified the internet as a communications tool and have developed specialised mailing lists such as Acad-AE-Med and Emed-L. 
WHAT'S IN THE INTERNET FOR A\&E MEDICINE? Accident and emergency medicine

The internet has the potential to become the place where day to day discussion on emergency medicine topics will take place. The "information footpath" as it is at present is rapidly becoming the "information superhighway" that it undoubtedly has the potential to become. Technology is now available for A\&E doctors from any number of institutions to interact simultaneously over the internet. They can share ideas, problems, and research on a one to one basis and also on a global level, by posting messages to newsgroups and mailing lists.

The availability of information and the speed of access to this information are the two greatest assets that the internet has to offer $A \& E$ medicine. The ease of access and relative affordability are particularly valuable. However, ignorance, and an inappropriate perception that advanced computer literacy is a prerequisite, could retard the development of the internet in $\mathrm{A} \& \mathrm{E}$ medicine.

\section{Accident and emergency trainees}

Several sites on the world wide web archive their case of the week. Other resources include ECG of the week, $x$ ray of the week, and clinical photographs. Archiving of these cases enables production of a tailored textbook of emergency medicine according to the needs of individual trainees. Book marking relevant WWW sites on one's browser or downloading relevant pages for reviewing off-line are two effective methods of accessing important educational material. Multimedia facilitates audible reproduction of croup and epiglottis, in addition to simulated auscultations. The internet provides both a textbook and a video library for $A \& E$ medicine, which can be continuously upgraded. It is possible, for instance, to download video footage of a percutaneous tracheostomy which can be catalogued on one's own computer.

A great number of North American emergency departments now have WWW sites, as do an increasing number of Australian and UK departments. Doctors wishing to pursue fellowships in emergency medicine can use the internet as an advertising notice board. ${ }^{2}$ Many sites on the internet offer information on toxicology and on paediatric and emergency medical services fellowships. The "hospital web site" allows access to information about a number of $A \& E$ departments and resources available within these departments.

Many sites are archiving medical material of major interest to $\mathrm{A} \& \mathrm{E}$ trainees. For instance, DESIRE (development of a European service for information on research and education) has established an orthopaedic information server which is in the process of scanning 60000 orthopaedic slides and publishing them on the internet. PARACELSE is a server in clinical toxicology developed at the University of Grenoble which includes protocols for the management of over 100 poisonings. It is updated weekly by a multidisciplinary team of toxicologists. MIDRIB (medical images digitised reference information bank) is a joint project funded between St
George's Hospital Medical School and Bristol University and launched early in 1997. It provides a comprehensive collection of medical images for use in teaching and research.

\section{Continuing medical education in $A \mathcal{E} E$ medicine} The internet has brought a new electronic information delivery system to $\mathrm{A} \& \mathrm{E}$ doctors. ${ }^{3}$ Until recently continuing medical education (CME) has been provided by paper journals, textbooks, and medical conferences. A wide range of CME is available to the $A \& E$ doctor, which is convenient, affordable, and accessible at the click of a button. Doctors can use a variety of usenet newsgroups to update on areas of advanced emergency medicine. Various WWW sites offer information about emergency systems and the organisation of emergency medical services in disaster areas and third world countries. Others link to many sites with $A \& E$ relevance such as trauma, emergency medical services, and paediatric critical care.

Several learned societies relevant to $A \& E$ have their own WWW sites. It is possible for doctors to browse through a calendar of upcoming events and conferences around the world and register for attendance.

Telemedicine is another resource providing CME on the internet. This can be particularly useful for $A \& E$ doctors based in rural areas, who can arrange to hold an interactive meeting with other colleagues at remote sites at a prearranged time. Such CME programmes have recently been described by a group of rural general practitioners in Wales. ${ }^{4}$

\section{Academia and research in $A \mathcal{E} E$ medicine}

The electronic journals homepage allows access to a great number of medical journals relevant to $A \& E$ medicine on the day of publication. ${ }^{5}$ These include the British Medical Fournal, the fournal of the American Medical Association, and the Annals of Emergency Medicine. This is of particular benefit to doctors working overseas, where journals may take many weeks to arrive by conventional mail. At present no journal publishes its entire contents on the internet. Most journals publish only a list of contents or the text of leading articles. There is a copyright issue regarding electronic journals which remains largely unresolved, although the British Library is currently undertaking research in this field.

Publishing on the internet is not without problems. While undoubtedly there is an advantage in the early and free dissemination of A\&E literature, the possibility of publications which have not been peer reviewed being published on the internet is a real and dangerous prospect. Benefits include the reduction of paper usage as a cost saving, the continued exposure of a journal both nationally and internationally, and bypassing difficulties experienced by quality journals not yet recognised by Medline. Issues of quality and regulation are being addressed as the development of electronic journalism continues. This will hopefully help in maintaining the prestige and recognition of current paper journalism. The potential loss of income by journals from a 
decrease in subscriptions is another real concern, although payment by credit card over the internet linked to password access may provide a financial solution.

The possibility of collaborative research has been made easier by the internet. Collection of data and downloading it to one designated site is now a much easier option. Data entered into user friendly electronic surveys at selected A\&E departments eliminates geographical separation as a barrier to conducting multiple site studies. Electronic collection of data can allow real time statistical processing and ongoing depiction of study progression.

Sharing of information between like minded $A \& E$ physicians has been made even easier by Acad-AE-Med, an academic A\&E mailing list. This list is aimed at fostering research and development within $\mathrm{A} \& \mathrm{E}$ in the United Kingdom, although some members contribute from other countries throughout the world.

The recently established Society of the Internet in Medicine is considering forming a subcommittee to advance the specialty further on the internet, particularly among European colleagues. Formal academic decisions will be made at MEDNET 97-the World Congress of the Internet in Medicine in Brighton.

\section{Prehospital care}

At a prehospital level there are many sites on the internet where paramedics, ambulance technicians, emergency medicine volunteers, and first aid workers can share experiences and communicate with each other on a worldwide basis.

\section{Accident and emergency nursing}

The internet acts as a forum for nurses working in emergency medicine to interface with colleagues on a national and international level. The emergency nursing homepage offers a good source of relevant internet material.

\section{Medical students}

The internet, and the WWW in particular, can be a cost-effective system of delivering $A \& E$ medical education to undergraduates. Overseas emergency departments regularly advertise their facilities, which helps students arrange electives. Undergraduates can now avail themselves of various tutorials in emergency medicine on the internet. Several sites provide links to case scenarios in paediatric emergency care, radiology, and toxicology. Case of the week, cases from grand rounds, $x$ ray of the week, and ECG of the week are other popular sites. Clinical photographs published on the internet are reproducible with excellent quality.

Continuously improving the quality of undergraduate medical education requires combining new technologies with established teaching methods. Some universities have now taken the initiative in providing courses for medical students on the internet. ${ }^{6}$

\section{The community}

Access to medical information in the home is unprecedented using the internet, and in the future the public may be better informed than ever on the management of common and eso- teric medical emergencies. A recent case report documented how a patient self diagnosed a deep vein thrombosis from the internet and self presented to his local A\&E department. Following a brief admission he was discharged with a diagnosis of superficial thrombophlebitis only to return four days later with frank haemoptysis and a pulmonary embolus. ${ }^{7}$

CURRENT VALUABLE MAILING LISTS, WEB SITES, AND NEWSGROUPS

Subscribing to mailing lists relevant to $A \& E$ medicine only takes a few clicks of a mouse button, as does unsubscribing if one receives too much mail. Examples of popular mailing lists include lists on adult emergency medicine, critical care medicine, paediatric emergency medicine, paediatric intensive care, wilderness medicine, trauma, toxicology, and disaster medicine. The membership of E-MED-L, the emergency medicine discussion group, exceeds 1000 .

Currently the University of Texas Health Science Center's Department of Emergency Medicine, in cooperation with a number of agencies, residencies and companies, maintains a homepage on the internet with the intention of putting the world of emergency medicine at one's fingertips. They have thoroughly embraced the new electronic super highway and through their home page it is possible to share ideas and problems on all aspects of $A \& E$ medicine with colleagues all over the world.

Various other sites on the WWW share information relevant to $A \& E$ medicine. For instance ACCRI (anaesthesia and critical care resources on the internet) is a weekly updated web site which provides a list of electronic resources of interest to anaesthesiologists and related specialties.

Louisiana State University Medical Center hosts TraumaNET. ${ }^{8}$ This server acts as a link to many other sites and as a navigational aid on the internet for trauma providers to sites covering all aspects of trauma care. There is a databank of interesting patient management cases in trauma and critical care.

The Orthopaedic Links page maintained by Clough in Ontario has 500 links in 20 different headings covering all aspects of acute and nonacute orthopaedics relevant to $A \& E$ medicine. At present it is in a growth phase and is expanding rapidly.

Other sites provide access to a directory of associations in emergency medicine. There is a list of emergency medical residency programmes in the United States. There are worldwide morbidity and mortality conferences and a site with information on the availability of research grants. Further information is available on prehospital care, legal aspects of emergency medicine, and politics in emergency medicine. There is a new emergency medicine electronic journal, GEMA, specific to the internet. Conference organisers post details of forthcoming conferences and it is possible to read about the 1998 International Conference on Emergency Medicine to be held in Vancouver, Canada.

There are various usenet newsgroups where topics of $A \& E$ relevance can be discussed. 
Most of these are under the sci. ${ }^{\star}$ category including sci.med.radiology and sci.med. orthopaedics.

\section{SECURITY ON THE INTERNET}

The security of information transmitted on the internet is suboptimal and there are no guarantees about confidentiality when dealing with individual cases. The accuracy of documents distributed on the internet currently relies on the integrity of those individuals posting them. $A \& E$ doctors who provide information on the internet should be aware that the legal situation surrounding publishing on the internet is largely unresolved.

The NHS is having to address this problem in developing a network for linking hospitals throughout the United Kingdom. It is recommended that $A \& E$ departments wishing to access the internet should do so with a stand alone computer and not with one linked to the department's computer system or the hospital network. A\&E doctors using the internet should be aware of the Data Protection Act.

WHAT IS THE FUTURE OF THE INTERNET IN A\&E MEDICINE?

There have been many speculations about the future of the internet in medicine which are applicable to A\&E medicine. We may for instance soon see the demise of journals as we know them, although some restrictions will inevitably be placed on the transmission of unrefereed and non-peer-reviewed medical reports. Plans already exist for the introduction of electronic peer review.

Communications systems will undoubtedly develop further and the role of the internet in $A \& E$ medicine, education, research, and clinical practice is set to expand. It is likely, given current trends, that the numbers of individual and corporate participants on the internet will continue to rise. It can be expected, with the introduction of the NHS web, that all $A \& E$ departments in the United Kingdom will soon have their own home pages.

The internet will enable rapid dissemination of information from responsible bodies such as the British Association for Accident and Emergency Medicine and the Faculty of Accident and Emergency Medicine. Guidelines and statement papers will be published on the internet and will be easily accessed as all $A \& E$ departments become computerised. Medline, available freely on the internet, is easily accessed for the latest information in the practice of evidence based medicine.

There is little doubt that this major advance in communications is here to stay. Its use in the world of $\mathrm{A} \& \mathrm{E}$ medicine as an education tool is set to go from strength to strength, and there is a strong argument to be made for those of us in $\mathrm{A} \& \mathrm{E}$ medicine to adopt the internet in everyday practice. It is unlikely to go away, so the sooner it is adopted by all involved in $A \& E$ medicine the greater is the likelihood of its potential being realised.

\section{Part II-What do I need to access the internet?}

The internet is now available to anyone with a personal computer, a modem, and a telephone line. It is owned by no one person or group; however, controllers of various networks which allow access to the internet usually command a fee for providing a service.

For the home user the prerequisites for accessing the internet are a telephone line, a computer, a modem, and an account with a service provider

A modem is a small box which is connected to the computer and the telephone line. Modems come in various speeds commonly known as 9.6, 14.4 , and 28.8 kilobaud, referring to the speed at which messages can be transmitted. The higher the number the faster the transmission. Any personal computer will access the internet, but from a practical viewpoint anything less powerful than a 386 is not worthwhile.

To link to the internet it is necessary to access and have an account with a service provider. Service providers regularly advertise in computer magazines, or friends who are on the internet may be able to recommend their own service provider. They may charge for the length of time you use their service or they may levy a standard monthly charge for their service. They supply the necessary software to get started and usually offer an excellent back up technical service to advise on teething problems. The service provider will supply you with an address which becomes your personalised address on the internet. It will usually incorporate the address of the service provider and allow you to choose your own identity and protected password for entry into an account.

Many hospitals have links to academic institutions or universities which have their own server. It is becoming increasingly possible for institutions that are involved with medical education to link up with their host university's server at little or no cost.

WHAT RESOURCES CAN IT PROVIDE?

The principal components of the internet include the world wide web, email, newsgroups, FTP (file transfer protocol), Telnet, and Gopher.

\section{World wide web}

The world wide web is a system which connects millions of computers worldwide and allows them to share information and media stored on these computers. The web part describes the links that exist between a computer and available resources such as documents, graphics, and photographs. Undoubtedly it is the advent of the world wide web that has led to the phenomenal interest in the internet. It has removed the difficulties experienced with basic and advanced computer terminology, allowing one easy access to other people's work published on the web. Access to the web is achieved through a software system known as a browser supplied by the service provider, and is as easy as point and click with a mouse. The initial page one sees on clicking onto the web browser is termed the homepage. Typing in the 
address of a web site known as the uniform resource locator (URL) on the browser's homepage takes you directly to that site or homepage. A good WWW browser such as Netscape or Internet Explorer incorporates all the features of email, the WWW, newsgroups and FTP in one simple integrated package.

email

Use of email allows for the almost instant exchange of electronic messages to an $A \& E$ colleague anywhere in the world who has an account on the internet. Software provided by the service provider allows you to log in at the click of a key to the host computer, that is, the service provider's computer. All one needs to do to send an instant message is to type the message, the destination email address, and press enter. In addition to a message, a previously prepared document or graphics can be sent with the message as an attachment.

Mailing lists are systems of sending one message to many people simultaneously. They contain members who have declared a particular interest in that list. Mailing lists are managed by listservers and it is necessary to subscribe (without charge), initially by sending a message to the listserver requesting inclusion in the list. Subsequently one receives an email of all messages sent to that list by contributors.

\section{Nezwsgroups}

Newsgroups are forums where people with a common interest can share information using a special newsreader software. Notices can be placed on a newsgroup, termed posting, using a software programme such as Usenet. This software is readily supplied by one's service provider. The advantage over mailing lists is that it allows participants to decide which articles they want to read and when. Newsgroups provide one of the best ways of sharing clinical problems with others on a worldwide basis. The possibilities have been dramatically demonstrated by the recent case of a 21 year old Chinese student. A friend posted a message about her acute illness on the internet which led to over 400 replies and a consensus that she had thallium poisoning. This was confirmed following the appropriate investigations and she improved with treatment. ${ }^{9}$

File transfer protocol

FTP involves moving files from one computer to another over the internet. It allows for trans- fer of documents, photographs, and even computer software from thousands of sites on the internet.

\section{Telnet}

This is a communications protocol that allows access to the resources of remote computers, often at libraries and universities. It also enables one to access email from a remote computer terminal while away from home or abroad.

\section{Gopher}

This is an information browser which allows searching of databases, reading of text files, and downloading of files to a user's hard drive.

\section{CONCLUSIONS}

Several textbooks are readily available in most bookstores, which take one through accessing the internet step by step. ${ }^{10}$ The rapid growth of the internet is being paralleled by the expansion in internet software which is making access easier. The difficulties which beginners have experienced over the last few years in accessing the internet have now largely been resolved and most people with just a basic knowledge of computers can be up and running on the internet within a matter of hours.

It should be noted that the internet is a dynamic technology which is constantly developing new techniques and expanding resources. Some of the comments in this paper, while valid at the time of writing, may not be valid when going to press as the internet continues to stride forward in the area of $A \& E$ medicine. In particular the uniform resource locators given below may change, a timely reminder that for all its benefits the internet lacks permanence.

1 Baldock C. Experience of a local hospital with the Internet: Brighton Health Care NHS Trust WwW pages. Mednet 96-European congress of the Internet in Medicin Brighton, October 1996.

2 Hart RG, Barbosa J. Emergency medicine residency brochure on the Internet. Acad Emerg Med 1996;3:386.

3 Pallen M. Guide to the Internet. BMJ 1995;311:1626-30.

4 Groves-Phillips S, Wynn-Jones J. Telemedicine and the Internet: their role in continuing medical education. Internet: their role in continuing medical education Mednet 96 European Congress of the Internet in Medicine. J Med 1995;333:1709-10.

6 Vanzyl AJ, Cesnik B. The Internet and its role in teaching 6 Vanzyl AJ, Cesnik B. The Internet and its role in teaching medical informatics to undergraduates. Medinfo

7 Ryan J. A clot in the system. Emerg Med 1996;8:188.

8 Block EFJ, Mire EJ. Trauma on the Internet: early experience with a world wide web server dedicated to trauma and critical care J Trauma 1996;41:265-70.

9 Craft N. No touch technique. BMJ 1996;312:318-9.

10 McKenzie B. Medicine and the Internet. Oxford: Oxford University Press, 1996.

\section{Appendix}

\section{Mailing lists relevant to $\mathbf{A} \& \mathbf{E}$}

Acad-ae-med: academic accident and emergency medicine discussion group mailbase@mailbase.ac.uk

Emerg-L: Adult emergency medicine discussion group. Listserv@VM.Marist.edu

Ped-Em-L: Paediatric emergency medicine discussion group

Listserv@brownVM.brown.edu

CCM-L: Critical care medicine. Listserv@UBVM.cc.Buffalo.edu.

Emed-L: Adult emergency medicine discussion group. Listserv@itsa.ucsf.edu

To subscribe, send a message to the address, typing: "subscribe "list name' (your name)" in the body of the message 
Newsgroups of A\&E relevance

sci.med.emergency

sci.med.cardiology

sci.med

alt.disasters.misc

sci.med.orthopedics

sci.med.radiology

misc.emerg-services

Homepages and URLs with A\&E relevance

The BMJ: http://www.bmj.com/

Wilderness medicine: EMS http://www.wemsi.on.ca/

The Hospital Web: http://neuro_www.mgh.harvard.edu/hospitalweb.nclk

Emergency medicine residencies: http://www.uth.tmc.edu/uth_orgs/emer_med/bookmarl.html

Prehospital care sites: http://rmstewart.uthscsa.edu/emergmedsites.html

Medline: http://www.healthgate.com/HealthGate/gate/free/

American College of Emergency Physicians: http://www.acep.org/

Emergency nursing: http://www.hooked.net/ ttrimble/enw/index.html

GEMA: http://gema.library.ucsf.edu:8081/

Trauma homepage: http://rmstewart.uthscsa.edu/

The University of Arizona Center for Research: http://www.ahsc.arizona.edu/aemrc/aemrc1.shtml

Vancouver international conference: http://www.interchange.ubc.ca/emerg_vh/7th.html

First aid online: http://www2.vivid.net/ cicely/safety/

Emergency medical services and nursing links: http://www.cris.com/ seaox/nurse.html

ECG interpretation: http://www.med.ufl.edu/medinfo/baseline/HN117.html

Orthopaedics link page: http://www.netshop.net/ cloughs/orthlink.html

PARACELSE: http://alpesmed.ujf-grenoble.fr/paracelse/paracelse.html

ACCRI: http://www.eur.nl/FGG/ANEST/wright/index.html

\section{Alison Gourdie Memorial Scholarship}

Applications are invited for the Alison Gourdie Memorial Scholarship. This award, to the value of $£ 1250$, is made annually to facilitate travel and/or research in the furtherance of emergency medicine. It is open to training grade doctors and consultants within five years of their appointment, and to any occupational group involved in the delivery of emergency care.

Application forms are available from the Faculty of Accident and Emergency Medicine, The Royal College of Surgeons of England, 35 Lincoln's Inn Fields, London WC2; tel 0171405 7071. Closing date for applications 31 October. 